\title{
Influencia de las competencias parentales en la atención y la flexibilidad cognitiva de escolares
}

\author{
Influence of Parenting Skills on Attention and Cognitive Flexibility in Schoolchildren
}

\author{
Francisca Bernal-Ruiz ${ }^{\mathrm{a}, *}$, Camila Paz Torres Pérez $z^{\mathrm{a}}$, Daniela Morin Cárdenas Tapia ${ }^{\mathrm{a}}$, Daniela Alejandra \\ Riveros Farías a , Camila Vilches Carvajal ${ }^{a}$, María Paz Farías Hurtubiaa ${ }^{a}$ Linda Kim Quintana López ${ }^{\mathrm{a}}$ \\ aUniversidad de Valparaíso, Chile
}

\section{Resumen}

Antecedentes: pese a que existe evidencia sobre el efecto que tienen los contextos en que se desenvuelven los/as niños/as en su desarrollo cognitivo y funcionamiento ejecutivo, hay pocos estudios que analicen la influencia específica que tienen determinadas competencias parentales en la atención y la flexibilidad cognitiva. Objetivo: el objetivo de este estudio fue determinar la influencia de las competencias parentales vinculares, formativas, reflexivas y protectoras, sobre la atención y la flexibilidad cognitiva de escolares de primer grado. Método: participaron 96 díadas de padre/madre/cuidador e hijo/a de escuelas chilenas. Los primeros fueron evaluados/as mediante la Escala de Parentalidad Positiva (e2p) y los/as niños/as con las subpruebas Sendero Color y Sendero Gris de la Batería ENFEN. Se realizaron análisis de correlación de Pearson y ANOVA unidireccional. Resultados: los resultados evidenciaron una influencia significativa de las competencias parentales formativas y protectoras en la atención de los/as niños/as. No se encontró influencia de ninguna competencia parental en la flexibilidad cognitiva. Conclusiones: estos resultados podrían explicarse desde un punto de vista madurativo, pues el desarrollo de la flexibilidad cognitiva está modulado por la adquisición previa de otras habilidades cognitivas y, en consecuencia, se desarrolla en etapas posteriores respecto al grupo etario de la muestra.

Palabras claves: competencias parentales; atención; flexibilidad cognitiva; escolares.

Para citar este artículo:

Bernal-Ruiz, F., Torres, C. P., Cárdenas, D. M., Riveros, D. A., Vilches, C., Farías, M. P. y Quintana, L. K. (2021). Influencia de las competencias parentales en la atención y la flexibilidad cognitiva de escolares. Liberabit, 27(2), e471. https://doi.org/10.24265/ liberabit.2021.v27n2.06

* francisca.bernal@uv.cl
Abstract

Background: Although there is evidence of the effect of the contexts in which children develop on their cognitive development and executive function, there are few studies that analyze the specific influence of certain parenting skills on attention and cognitive flexibility. Objective: This study aimed to determine the influence of bonding, formative, reflective and protective parenting skills on attention and cognitive flexibility in first grade schoolchildren. Method: Ninety-six (96) dyads of fathers/ mothers/caregivers and children from Chilean schools participated in the study. The former were evaluated with the Positive Parenting Scale (e2p) and the latter with the subtests color path and gray path of the Neuropsychological Assessment of Executive Functions in Children (ENFEN). The data was analyzed using Pearson's correlation coefficient and one-way ANOVA. Results: The results showed a major influence of formative and protective parenting skills on children's attention. No influence of any parenting skills on cognitive flexibility was found. Conclusions: These results could be explained from a maturational point of view since the development of cognitive flexibility is modulated by the previous acquisition of other cognitive skills. Consequently, it is developed in later stages with respect to the age group of the sample.

Keywords: Parenting skills; attention; cognitive flexibility; schoolchildren.

Este es un artículo Open Access publicado bajo la licencia Creative Commons Atribución 4.0 Internacional. (CC-BY 4.0)

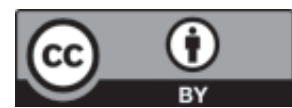

Universidad de San Martín de Porres, Lima - Perú http://ojs3.revistaliberabit.com 


\section{Competencias parentales y su impacto en el funcionamiento ejecutivo en la infancia}

La relación que existe entre los/as niños/as con sus padres, madres o cuidadores, resulta de gran relevancia para el campo de la psicología. Se ha evidenciado que el impacto que tiene el contexto en el que se desenvuelven los/as niños/as durante sus primeros años de vida es muy significativo en su desarrollo cognitivo y socioafectivo (Velarde \& Ramírez, 2017).

En efecto, la teoría ecológica de Bronfenbrenner (1979) destaca la importancia del entorno familiar inmediato y otros elementos del ambiente en el desarrollo individual; en donde no solo se enfatizan los factores ontogenéticos, sino también los contextuales (Vargas-Rubilar \& Arán-Filippetti, 2014). Sin duda, uno de los contextos sociales más importantes es el de la familia, especialmente en la infancia, en donde una de las funciones principales de los padres, madres o cuidadores es entregar la estimulación adecuada que permita a los/as hijos/as desarrollarse y relacionarse de manera efectiva en otros entornos y con otras personas. Dicho de otro modo, la influencia del contexto familiar en esta etapa es sinónimo de saberes ligados a valores, normas y costumbres; ya que la manera en cómo la familia brinda estos conocimientos influirá en la adquisición de habilidades tales como la resolución de problemas, conductas prosociales y regulación emocional (Martínez, 2010).

En esta misma línea, diversos autores (Caascango et al., 2020; Franco-Nerín et al., 2014; Rojas, 2020; Treat et al., 2019) coinciden en que los padres modulan las conductas de los/as hijos/as en la dirección que ellos valoran y desean. Asimismo, el aprendizaje generado a través de esta «modulación de conductas» se encuentra marcado tanto por los estilos de crianza (autoritario, permisivo o democrático) propuestos por Baumrind (1967) como por las competencias parentales (CP) propuestas por Gómez y Muñoz (2014), las que incluyen las competencias formativas, reflexivas, vinculares y protectoras, que a su vez influyen en el desarrollo evolutivo del/de la menor.
De acuerdo con estos autores, las CP vinculares corresponden a prácticas cotidianas de parentalidad que tienen como objetivo promover un estilo de apego seguro y un desarrollo socioemocional adecuado en los/as niños/as. Por otro lado, las competencias formativas son conocimientos parentales relacionados con acciones que promuevan el desarrollo, la socialización y el aprendizaje de los/as niños/as. Las competencias reflexivas son aquellas actitudes de los padres, madres o cuidadores que buscan promover la reflexión en torno a la trayectoria del ejercicio propio de la parentalidad, implican una revisión de las prácticas parentales actuales y un monitoreo del desarrollo de los hijos e hijas, con el objetivo de contar con una retroalimentación en relación con las otras áreas de la parentalidad. Finalmente, las competencias de tipo protectoras corresponden a aquellos conocimientos cotidianos de la parentalidad, los cuales tienen como fin resguardar y cuidar de manera adecuada a los/as hijos/as, protegiendo de esta forma, sus necesidades de desarrollo humano, respetando sus derechos y procurando velar por su integridad física, mental y sexual (Gómez \& Muñoz, 2014; Vera \& Apolo, 2020).

De esta manera, estas CP propuestas por Gómez y Muñoz (2014) influyen en el desarrollo cognitivo, que va ligado a los conocimientos, habilidades y funciones que el/la niño/a va adquiriendo en relación con su crecimiento, y también a los procesos psicológicos básicos; todo esto a la base de sus interacciones primarias, desde su familia como entorno más cercano hasta sus pares insertos en contextos escolares y de ocio. Esto permite identificar la importancia de brindarle a los/as niño/as espacios en donde se generen vínculos positivos y aprendizajes óptimos en beneficio de su desarrollo integral (García et al., 2019). De esta idea deriva el concepto de parentalidad, la cual se manifiesta en las distintas acciones llevadas a cabo por padres, madres o cuidadores durante el proceso de crianza, cuidado y educación de sus hijos/as y el que no solo abarca aspectos biológicos, sino también psicosociales (Bornstein, 1995; Vargas-Rubilar \& Arán- Filippetti, 2014). Además, la parentalidad se enfoca en los 
conocimientos, creencias y comportamientos que tienen los padres, madres o cuidadores en relación con distintos aspectos de la vida del/la niño/a, tales como la salud, su nutrición, la importancia de la estimulación, el aprendizaje, y la relevancia de los espacios tanto físicos como sociales en donde se desenvuelven (De Cock et al., 2017).

Hughes y Ensor (2009) hallaron en sus estudios que distintos factores asociados al ámbito familiar, como el apoyo en la ejecución de actividades ligadas a metas específicas, la buena organización, las prácticas parentales positivas y estables, favorecen el buen funcionamiento ejecutivo de los hijos e hijas, los que podrían ser capaces de resolver situaciones complejas, establecer determinados objetivos y prever las consecuencias de sus actos. A su vez, varios estudios destacan que el cuidado, la estimulación y el buen trato por parte de los progenitores a sus hijos/ as son esenciales para el desarrollo, la organización y el funcionamiento del cerebro en los/as infantes (Barudy \& Dantagnan, 2010; De Cock et al., 2017). Por ejemplo, Schroeder y Kelley (2010) en su estudio encontraron que la estructura familiar, los límites y la protección establecidos por los padres, madres o cuidadores se asocian de manera significativa con el desarrollo cognitivo y más específicamente con las funciones ejecutivas (FE) de los/as niño/as, particularmente con aspectos vinculados a la planificación, memoria de trabajo, organización, inhibición, monitoreo, y control emocional.

Las FE son un constructo que en las últimas décadas ha sido ampliamente estudiado, principalmente, porque se ha logrado evidenciar que sus mecanismos se ponen en marcha en una vasta variedad de situaciones y etapas vitales, por lo que son indispensables para el desarrollo óptimo y socialmente adaptado de todo ser humano (Lezak, 2004). Son múltiples las definiciones que se han planteado acerca de las FE, las que generalmente son entendidas como habilidades cognitivas de orden superior que abarcan y dirigen procesos necesarios para llevar a cabo comportamientos dirigidos hacia objetivos complejos, los cuales requieren de un manejo creativo ante situaciones novedosas en las que su función se basa en generar, controlar, ejecutar y reajustar la conducta según las exigencias del medio en que se presentan (Verdejo-García \& Bechara, 2010).

Existen otras definiciones de FE, por ejemplo, Tirapu-Ustárroz et al. (2018) señalan lo siguiente:

Son un conjunto de procesos implicados en la resolución de situaciones novedosas y que nos permiten actuar de manera flexible en entornos cambiantes basándonos en la anticipación y la predicción para reducir la incertidumbre del entorno en base a nuestra adaptación biológica, personal y social. (p. 1)

A su vez, otros autores como Miyake et al. (2000), Diamond (2013) y Diamond y Ling (2020) plantean que el funcionamiento ejecutivo se refiere a un conjunto de capacidades cognitivas diferentes entre sí, pero permanentemente interrelacionadas, las cuales participan en el comportamiento flexible dirigido a objetivos y que generalmente son categorizadas en tres componentes básicos: memoria de trabajo, flexibilidad cognitiva y control inhibitorio. De esta manera, pese a las múltiples definiciones que engloban al concepto, se logran distinguir estos tres componentes principales, de los cuales se desprenden a su vez, el razonamiento de problemas, la planificación y la resolución de conflictos (Bernal-Ruiz et al., 2018).

En este estudio, nos enfocaremos específicamente en el dominio ejecutivo de la flexibilidad cognitiva y estudiaremos la atención que si bien, no ha sido señalada como un factor principal en el modelo explicativo del funcionamiento ejecutivo, se considera una habilidad cognitiva que modula el desarrollo de los tres componentes principales de las FE (TirapuUstárroz et al., 2018).

La flexibilidad cognitiva (FC) refiere a la habilidad para adaptar nuestros pensamientos y conductas en situaciones cambiantes y lograr darnos cuenta de que si algo no está resultando como se espera o dejó de 
funcionar, podemos reajustar nuestra conducta y pensamientos para lograr el objetivo propuesto y, así, lograr adaptarnos al entorno y a nuevas situaciones que lo demanden (Diamond, 2013). En este sentido, la FC es la que permite al individuo crear una nueva conducta mejorada y adaptativa, pasar a nuevas actividades, generar cambios en las rutinas, aprender de los errores, elaborar estrategias y alternativas, brindar soluciones a situaciones novedosas, organizar ideas. En el ámbito escolar permite, por ejemplo, pasar de la suma a la resta y aprender la diferencia entre números y letras, entre otras cosas (Caascango et al., 2020).

De acuerdo con el desarrollo evolutivo de las FE, la FC se desarrolla más tardíamente que el control inhibitorio y la memoria de trabajo (Davidson et al., 2006; Diamond, 2013), a partir de lo cual se podría inferir que requiere de las demás FE. Diamond (2013) afirma que, para lograr ejecutar un cambio, se tiene que previamente inhibir la perspectiva anterior y cargar en la memoria de trabajo una perspectiva diferente.

Por otro lado, respecto a la atención, según Luria (1984) esta es la responsable de extraer los elementos esenciales para la actividad mental y es el proceso que mantiene una estrecha vigilancia sobre el curso de la actividad mental. Smolak et al. (2020) afirman que los componentes de la atención incluyen lo siguiente: 1) la alerta, ligada a la vigilancia y preparación de la atención para ser utilizada para el procesamiento, que incluye tanto el componente tónico (durante intervalos de tiempo más largos), como fásico (durante períodos breves < 1 segundo); 2) la orientación, la cual tiene que ver con la atención dirigida a un estímulo en el espacio externo o interno; 3) la atención selectiva, la cual corresponde a la selección de un objetivo específico con exclusión de los distractores con el fin de mejorar el procesamiento; y 4) la atención sostenida, relacionada al mantenimiento de la atención y la vigilancia por un largo periodo de tiempo.

Por otro lado, la atención ha sido considerada como una propiedad de los sistemas funcionales, puesto que las características que poseen tanto la atención selectiva como la sostenida, activan estos sistemas funcionales y mantienen su activación mientras se incorporan y procesan estímulos e información (Rebollo \& Montiel, 2006; Ricker et al., 2018). En lo que se refiere a la estructura funcional, se pueden considerar las funciones ejecutivas, las funciones cognitivas y las funciones que colaboran en su organización. Estas últimas son la memoria del trabajo, la atención (selectiva y sostenida) y quizás la flexibilidad cognitiva, lo que difiere de la postura que incorpora a la atención, memoria de trabajo y flexibilidad cognitiva como funciones ejecutivas (Rebollo \& Montiel, 2006). En este sentido, el concepto de atención se superpone con el de FE, ya que usualmente se describen los sistemas atencionales como constructos multidimensionales que tienen varias subfunciones como las de regulación y vigilancia, el enfoque selectivo de la atención, la atención sostenida y la división de la atención (Fortenbaugh et al., 2017; Klenberg et al., 2001; Sui \& Rotshtein, 2019).

A la luz de los antecedentes anteriores, que reconocen la influencia directa del comportamiento de los padres, madres o cuidadores sobre el desarrollo del funcionamiento ejecutivo de sus hijos/as, surge el interés por averiguar qué habilidades parentales favorecen el desarrollo de la FC y la atención; ya que se sabe que los factores familiares facilitan el surgimiento de potencialidades no solo cognitivas, sino que también sociales y afectivas mediante el despliegue de competencias vinculares, formativas, reflexivas y protectoras que poseen las figuras de apego (Caascango et al., 2020).

Existen diversos estudios que vinculan las conductas por parte de los padres, madres o cuidadores con un mayor desarrollo de la atención y la FC de sus hijos/as. En este sentido, respecto a la atención, Ross et al. (2019) se propusieron examinar el papel que cumplen los episodios de estrés agudo en el rendimiento cognitivo de los/as niños/as a través de actividades de tipo GO/NO/GO. En su estudio se pudo observar que los/as niños/as expuestos a estrés agudo 
consiguieron un desempeño significativamente inferior en una medida de atención sostenida (ensayos GO) en relación con el estímulo estresante y a la posterior ejecución de la tarea. Por otra parte, estos autores no encontraron diferencias significativas en el desempeño del control inhibitorio (ensayos NO GO). De esta manera, este estudio advirtió que los/as hijos/as de madres que autoinformaron un mayor estrés en el periodo de crianza, presentaron una mayor vulnerabilidad a los efectos del estrés agudo en tareas del tipo GO/NO/GO que involucran atención sostenida. Considerando la edad promedio de la muestra de este estudio (5 años) es posible identificar que en esta etapa de la infancia se van adquiriendo habilidades que permiten tolerar la angustia y la frustración de una manera más autónoma, por lo que los/as participantes del estudio mostraron una dificultad para dirigirse a objetivos que involucraron atender de manera sostenida luego de experimentar episodios de estrés agudo. Además, el estudio concluye que los/as niños/as que tienen padres/madres o cuidadores con menos capacidades para hacer frente al estrés de una manera saludable o proporcionar un amortiguamiento social por medio de una respuesta sensible, tienen más dificultades para recuperarse luego de episodios de estrés agudo y para responder en tareas cognitivas (Ross et al., 2019).

En un estudio anterior realizado por De Cock et al. (2017), se concluyó que el vínculo de la madre permite predecir dificultades de funcionamiento ejecutivo en su hijo/a, asociación que estaba mediada por el estrés presente en el proceso de maternidad. En este sentido, el estrés de los padres fue el mediador entre la mala vinculación postnatal materna y paterna y el rendimiento ejecutivo de los/as niños/as, en donde posiblemente el estrés se manifiesta en un comportamiento negativo de los padres, lo que a su vez impacta en la interacción que tienen con su hijos/ as y que, por lo tanto, repercute en el desarrollo cognitivo de estos/as. Además, se señala que el cuidado parental temprano puede influir en el sistema de respuesta al estrés de los/as niños/as, dado que los niveles altos de estrés en el padre, madre o cuidador pueden generar un ambiente estresante en los/as hijos/ as y repercutir en sus propios niveles de estrés, afectando su desarrollo cognitivo. De esta manera, los padres, madres o cuidadores que poseen elevados niveles de estrés pueden pasar menos tiempo con sus hijos/as y participar en pocas actividades de estimulación con ellos/as. Asimismo, se puede generar un ambiente caótico y desorganizado marcado por el estrés, lo cual genera en los/as niños/as menos espacio para desplegar de manera óptima sus habilidades ejecutivas y atencionales (De Cock et al., 2017).

Respecto a la relación de las habilidades parentales y la FC de los/as hijos/as, destaca el estudio realizado por Muñoz-Gómez (2019) en el cual evaluó los estilos parentales y su relación con el desarrollo de la inhibición y la FC en niños/as de 12 años. Entre sus principales hallazgos encontró una relación moderada y negativa entre coerción y FC, entendiéndose como coerción el grado en que expresan los padres y madres su desacuerdo con los comportamientos que consideran inadecuados en sus hijos e hijas, e intentan eliminar dichas conductas a través de la imposición de restricciones y de la coacción verbal o física. Este resultado se ve corroborado por los hallazgos anteriores de Musso (2010), quien encontró que los niños de 4 y 5 años que sienten un mayor control hostil de sus padres (obteniendo puntuaciones altas en coerción) tienen una ejecución inferior en tareas de planificación, en las cuales se necesita control inhibitorio y FC.A la luz de estos antecedentes, es necesario preguntarse: ¿ ¿ienen las CP influencia sobre la atención y la FC de los/as niños/as en etapa escolar?, ¿Qué CP específicas tienen efecto sobre la atención y la FC de los/as escolares? En relación a lo anterior, se plantea como objetivo determinar la influencia de las competencias parentales (vinculares, formativas, reflexivas y protectoras) sobre la atención y la flexibilidad cognitiva de los/as escolares de la muestra. Adicionalmente, tomando en cuenta el corpus teórico actual sobre esta temática, se espera visualizar una influencia de las CP sobre el desempeño de la atención y la FC de los/as niños/as, que será estadísticamente significativa. Específicamente, se espera que las CP 
formativas tengan influencia tanto en la atención como en la FC de los/as niños/as; mientras que las CP vinculares influyan sobre el desarrollo de la FC de los/ as niños/as.

\section{Método}

\section{Participantes}

La muestra no probabilística y por conveniencia, incluyó 96 diadas de padres, madres o cuidadores e hijos/as reclutados/as en dos escuelas ubicadas en la ciudad de Valparaíso, Chile, una de dependencia pública $(N=51)$ y la otra de dependencia privada $(N=45)$. Los padres/madres/cuidadores que participaron corresponden a 19 hombres, cuya edad promedio era de 37.1 años, y 77 mujeres, con una edad promedio de 34.1 años. En el caso de los/as niños/as, participaron 39 varones, con una edad promedio de 6.79 años, y 57 mujeres, con una edad promedio de 6.77 años (ver Tabla 1). Por su parte, los/as niños/as debían cumplir un único criterio de inclusión, el cual era estar oficialmente matriculados en primer grado de las escuelas. Entre los criterios de exclusión de los/las niños/as se encontraban: a) presentar cualquier trastorno del desarrollo neurológico diagnosticado (TEA, TDAH, trastornos del habla y el lenguaje, trastornos motores, entre otros), b) estar bajo tratamiento psicofarmacológico o médico que pueda afectar el desempeño de los dominios de las funciones ejecutivas evaluadas, c) no tener desarrollada la habilidad de conteo hasta el 30, y d) estar en desacuerdo con la participación en el estudio.

\section{Instrumentos}

Escala de Parentalidad Positiva (e2p). Instrumento para evaluar las CP de los padres/ madres/cuidadores, creada por Gómez y Muñoz (2014), la cual consiste en un cuestionario autoadministrado que cuenta con 54 ítems. El cuestionario tiene como objetivo identificar las competencias parentales que aquellos utilizan en el cuidado cotidiano de su hijo/a o niño/a a su cargo.
Los 54 ítems describen prácticas habituales de crianza, que se dividen en 4 áreas, estas son vínculo, formación, protección y reflexión. Estas áreas contienen subescalas: competencias vinculares (14 ítems), competencias formativas (12 ítems), competencias protectoras (17 ítems) y competencias reflexivas (11 ítems). La manera de responder tiene cuatro opciones y se deben escoger entre Casi nunca, A veces, Casi siempre y Siempre. La aplicación de este cuestionario dura aproximadamente 20 minutos.

Para obtener el puntaje de las subescalas, es necesario que sean sumados los puntajes asociados a ellas y para obtener el puntaje de la Escala de Competencia Parental Total son sumados los puntajes de las 4 subescalas. Con este puntaje se puede acceder al percentil del evaluado/a. Finalmente, al interpretar los resultados, se deben distinguir entre 3 rangos o zonas de CP según dicho percentil, la «zona de riesgo» incluye los percentiles 10 y 20; la «zona de monitoreo» los percentiles 30 y 40, y la «zona óptima» los percentiles 50 o superior. En cuanto a sus propiedades psicométricas, la consistencia interna de la escala total reportada por los autores es $\alpha=.946$ de Cronbach, y para cada dimensión de la escala es la siguiente: a) vinculares $\alpha=.898$ de Cronbach, b) formativas $\alpha=.860$ de Cronbach c) protectoras $\alpha=.845$ de Cronbach, y d) reflexivas $\alpha=.817$ de Cronbach.

Batería de Evaluación Neuropsicológica de las Funciones Ejecutivas en niños/as ENFEN. Instrumento creado por Portellano et al. (2009) para evaluar el nivel de madurez y el rendimiento cognitivo en actividades relacionadas con las funciones ejecutivas en niños entre los 6 y los 12 años. Para este estudio se optó por la versión española de la batería y de esta se utilizó la Subprueba de Senderos que está compuesta por el Sendero Gris y el Sendero Color, que evalúan la atención y la FC, respectivamente, y que deben ser aplicadas en ese orden. Su aplicación es individual y bastante sencilla; por lo mismo, puede ser aplicada a distintos tipos de 
población, incluso aquellas con un nivel bajo de educación (Lozano et al., 2012).

Cada una de las dos partes de la subprueba de Senderos viene precedida por un ítem de entrenamiento para facilitar al sujeto evaluado/a la comprensión de la tarea y obtener así una evaluación más precisa de su habilidad. En la primera parte (Sendero Gris), la cual sirve para evaluar atención selectiva y focalizada, se pide al niño/a que una mediante una línea los números en orden descendente del 20 al 1 que aparecen ordenados aleatoriamente en una hoja (sendero 1). En la segunda parte (Sendero Color) que evalúa la FC, debe unir los números del 1 al 21 que aparecen ordenados aleatoriamente en otra hoja, pero alternando los que son de color rosado y los que son de color amarillo. En cuanto a las propiedades psicométricas de la batería ENFEN, esta fue validada en una muestra de 837 escolares de edad entre 6 y 12 años. Implementando un Análisis Factorial Exploratorio (AFE) a través del principal método de componentes, mostró una buena validez. Tanto KaiserMeyer-Olkin (KMO = .84) como Bartlett, en la prueba de esfericidad $(p<.001)$, indican una excelente adecuación de muestreo. Por su parte, respecto a la confiabilidad, el alfa de Cronbach de la escala total fue igual a .76; lo que se considera alto cuando se desarrollan medidas novedosas (Taber, 2018).

\section{Procedimiento}

En primer lugar, y una vez obtenidas las autorizaciones para realizar la investigación en las escuelas, se participó en cada una de ellas, en 2 reuniones de apoderados/as de los primeros básicos con la finalidad de explicar los objetivos de la investigación, firmar el consentimiento informado y aplicar la Escala de Parentalidad Positiva e2p a los padres, madres o cuidadores que aceptaron participar con sus hijos/as en la investigación.

Posteriormente, los/as estudiantes cuyos padres, madres o cuidadores autorizaron su participación, fueron evaluados/as individualmente durante la jornada escolar, en una sala especialmente habilitada que cumplía con las especificaciones estándar para la realización de evaluaciones cognitivas. Las evaluaciones tuvieron una duración en promedio de 25-30 minutos para cada niño/a. El periodo de evaluación de los/as niños/as se completó en 4-6 semanas, a un ritmo de aproximadamente 16 niños/ as por evaluadora. Por último, como retribución por participar en el estudio, se invitó a los padres, madres y cuidadores de los niños/as de la muestra a participar en un taller de parentalidad positiva.

\section{Análisis de datos}

Inicialmente, se llevaron a cabo análisis descriptivos para resumir la información demográfica de la muestra. Luego, se realizaron análisis de correlación bivariante de Pearson para estimar la presencia de una asociación entre las CP y el desempeño ejecutivo en tareas de atención y la FC de los/as niños/as. Finalmente, para evaluar la influencia de las CP en la atención y la FC de los/as niños/as se realizaron análisis ANOVA unidireccional para evaluar la existencia de diferencias significativas en atención y la FC de los/as niños/as según el nivel de CP de sus padres/madres/cuidadores (óptimo, monitoreo, riesgo). Todos los análisis se hicieron con el software estadístico Jamovi, versión 1.1.9.0 (The Jamovi Project, 2020).

\section{Consideraciones éticas}

Respecto a las normativas éticas, en esta investigación todos los procedimientos fueron implementados siguiendo los lineamientos de la Declaración de Singapur sobre la integridad en la investigación (Comisión Nacional de Investigación Científica y Tecnológica, 2010). Por lo mismo, fue definido un protocolo de consentimiento informado que debía ser firmado por los padres/madres/ cuidadores de los/as niños/as de la muestra. De esta manera, la investigación contó solo con la participación de los/as estudiantes que tenían sus consentimientos debidamente acreditados y firmados y que dieron su asentimiento. 


\section{Resultados}

\section{Análisis descriptivos}

\section{Tabla 1}

Muestra y descripciones demográficas de las díadas de padres/madres/cuidadores e hijos/as

\begin{tabular}{|c|c|c|c|c|c|}
\hline \multirow{2}{*}{\multicolumn{2}{|c|}{ Tipo escuela }} & \multicolumn{4}{|c|}{ Díadas $(N=96)$} \\
\hline & & \multicolumn{2}{|c|}{ Padres/madres/cuidadores } & \multicolumn{2}{|c|}{ Niños/as } \\
\hline Escuela privada & Sexo & Hombre & Mujer & Hombre & Mujer \\
\hline \multirow[t]{2}{*}{$N=45(46.9 \%)$} & & $N=9(20 \%)$ & $N=36(80 \%)$ & $N=24(53.3 \%)$ & $N=21(46.7 \%)$ \\
\hline & Edad & $40.8(4.65)$ & $37.8(4.33)$ & $6.75(.44)$ & $6.67(.48)$ \\
\hline Escuela pública & Sexo & Hombre & Mujer & Hombre & Mujer \\
\hline \multirow[t]{2}{*}{$N=51(53.1 \%)$} & & $N=10(19.6 \%)$ & $N=41(80.4 \%)$ & $N=15(29.4 \%)$ & $N=36(70.6 \%)$ \\
\hline & Edad & $33.3(3.20)$ & $29.3(5.83)$ & $6.87(.74)$ & $6.83(.61)$ \\
\hline
\end{tabular}

\section{Análisis de correlación entre las competencias de los padres/madres/cuidadores y la atención y flexibilidad cognitiva de los/as niños/as}

En primer lugar, se analizó el supuesto de normalidad univariante para el análisis de correlación de Pearson, el que se cumplió. Posteriormente, se obtuvo la matriz de correlación entre las CP (i.e., protectoras, vinculares, formativas y reflexivas) y las FE de los/as niños/as (i.e., atención y flexibilidad cognitiva). Se observan correlaciones significativas ( $p$ $<.01)$ entre la mayoría de las competencias parentales y la atención de los/as niños/as, que van desde $r=.200$ hasta $r=.361$. No ocurre lo mismo con la flexibilidad cognitiva, pues no se encontraron correlaciones significativas entre ninguna de las competencias parentales y dicha función ejecutiva (ver Tabla 2).
Diferencias en el desempeño ejecutivo de los/ as niños/as: análisis de la varianza (ANOVA unidireccional)

Las CP se clasificaron en términos categóricos (i.e., óptimas, de monitoreo y de riesgo) de acuerdo con las puntuaciones de corte de la Escala de Parentalidad Positiva (e2p) (Gómez \& Muñoz, 2014). De esta manera, se realizaron análisis ANOVA unidireccionales para evaluar el efecto de las competencias parentales en la atención y la flexibilidad cognitiva de los/as niños/as.

Los principales resultados muestran una influencia significativa tanto de las CP formativas como protectoras en la atención de los/as niños/as, con un tamaño de efecto de $\eta 2=.129$ y .115 , respectivamente, los que pueden ser interpretados como grandes según los criterios de Cohen (1988) (Ver Tabla 3). 
Tabla 2

Correlación de las competencias parentales con la atención y flexibilidad cognitiva de los/as niños/as

\begin{tabular}{|c|c|c|c|}
\hline Competencias parentales & & Atención & Flexibilidad cognitiva \\
\hline \multirow[t]{2}{*}{ Competencias parentales totales } & pearson's $r$ & $.337 * *$ & -.136 n.s. \\
\hline & $p$ value & $<.01$ & .906 \\
\hline \multirow[t]{2}{*}{ Competencias protectoras } & pearson's $r$ & $.300 * *$ & $-.098 n . s$. \\
\hline & $p$ value & $<.01$ & .786 \\
\hline \multirow[t]{2}{*}{ Competencias vinculares } & pearson's $r$ & $.200 * *$ & -.131 n. s. \\
\hline & $p$ value & $<.01$ & .898 \\
\hline \multirow[t]{2}{*}{ Competencias formativas } & pearson's $r$ & $.361 * *$ & -.133 n. s. \\
\hline & $p$ value & $<.01$ & .901 \\
\hline \multirow[t]{2}{*}{ Competencias reflexivas } & pearson's $r$ & $.267 * *$ & -.117 n.s. \\
\hline & $p$ value & $<.01$ & .872 \\
\hline
\end{tabular}

Nota. ${ }^{* *} p<.01 ;$ n. $s .=$ no significativo.

Tabla 3

Diferencias en los niveles de competencias parentales en función de la atención, la flexibilidad cognitiva y las dimensiones de las competencias parentales

\begin{tabular}{lccccccccc}
\hline & \multicolumn{7}{c}{ Dimensiones de las competencias parentales } \\
\cline { 2 - 8 } & \multicolumn{1}{c}{ CP protectoras } & CP vinculares & CP formativas & \multicolumn{2}{c}{ CP reflexivas } \\
\hline Funciones ejecutivas & $p$ & $\eta^{2}$ & $p$ & $\eta^{2}$ & $p$ & $\eta^{2}$ & $p$ & $\eta^{2}$ \\
Atención & $<.01$ & $.115^{\mathrm{a}}$ & .062 n. $s$. & .058 & $<.01$ & $.129^{\mathrm{b}}$ & $<.05$ & $.086^{\mathrm{b}}$ \\
Flexibilidad cognitiva & .216 n. s. & .032 & .251 n. s. & .029 & .567 n. s. & .012 & .269 n. s. & .028 \\
Tamaño promedio del efecto & - & .213 & - & .169 & - & .133 & - & .046 \\
\hline
\end{tabular}

Nota. a = las comparaciones post hoc muestran que las puntuaciones de los/as niños/as cuyos padres se encuentran en el nivel de riesgo son significativamente más bajas que las puntuaciones de los otros dos niveles (óptimo y monitoreo); b = las comparaciones post hoc muestran que las puntuaciones de los/as niños/as cuyos padres se encuentran en el nivel de riesgo solo son significativamente más bajas que las puntuaciones del grupo de nivel óptimo; n.s. = no significativo.

Las comparaciones post hoc de Tukey mostraron que el rendimiento atencional fue significativamente menor para los/as niños/as cuyas habilidades de protección y formativas de sus padres/madres/ cuidadores se clasificaron como «de riesgo» $(p<.01)$ (ver Tabla 4). Mientras que, en el caso de la flexibilidad cognitiva, al no existir una correlación significativa entre esta función ejecutiva y las competencias parentales, es posible identificar que tampoco se evidencia una influencia significativa de las dimensiones de las competencias parentales en la flexibilidad cognitiva. 
Tabla 4

ANOVA unidireccional para los efectos de las competencias parentales en la atención y flexibilidad cognitiva de los/ as niños/as

\begin{tabular}{|c|c|c|c|c|c|c|c|}
\hline & \multicolumn{3}{|c|}{ Competencias parentales (categórica) } & \multicolumn{3}{|c|}{ Desempeño ejecutivo niños/as } & \multirow[b]{2}{*}{$\begin{array}{c}\text { Tamaño Efecto } \\
\eta^{2}\end{array}$} \\
\hline $\begin{array}{l}\text { Dominio } \\
\text { ejecutivo }\end{array}$ & Dimensión & Nivel & $N$ & Media & DS & $p$-value & \\
\hline \multirow[t]{3}{*}{ Atención } & Formativa & Óptimo & 2 & 5.38 & 2.45 & $<.01 * *$ & .129 \\
\hline & & Monitoreo & 16 & 4.38 & 2.45 & & \\
\hline & & Riesgo & 8 & 3.45 & 2.14 & & \\
\hline \multirow[t]{3}{*}{ Atención } & Protectora & Óptimo & 27 & 5.18 & 2.33 & $<.01 * *$ & .115 \\
\hline & & Monitoreo & 14 & 5.78 & 2.83 & & \\
\hline & & Riesgo & 55 & 3.74 & 2.29 & & \\
\hline \multirow[t]{3}{*}{ Atención } & Vincular & Óptimo & 2 & 5.46 & 2.54 & .062 n.s. & .058 \\
\hline & & Monitoreo & 19 & 4.58 & 2.52 & & \\
\hline & & Riesgo & 55 & 4.00 & 2.34 & & \\
\hline \multirow[t]{3}{*}{ Atención } & Reflexiva & Óptimo & 41 & 5.12 & 2.53 & .015 n.s. & .086 \\
\hline & & Monitoreo & 22 & 4.64 & 2.24 & & \\
\hline & & Riesgo & 33 & 3.49 & 2.29 & & \\
\hline \multirow[t]{3}{*}{ Atención } & CP Totales & Óptimo & 30 & 5.47 & 2.57 & $<.01 * *$ & $.098^{\mathrm{a}}$ \\
\hline & & Monitoreo & 11 & 4.91 & 2.21 & & \\
\hline & & Riesgo & 55 & 3.30 & 2.22 & & \\
\hline \multirow[t]{3}{*}{ Flex. cognitiva } & Formativa & Óptimo & 42 & 2.86 & 2.45 & .57 n.s. & .012 \\
\hline & & Monitoreo & 16 & 3.56 & 2.42 & & \\
\hline & & Riesgo & 38 & 3.18 & 2.14 & & \\
\hline \multirow[t]{3}{*}{ Flex. cognitiva } & Protectora & Óptimo & 27 & 2.52 & 2.03 & .22 n.s. & .032 \\
\hline & & Monitoreo & 14 & 3.79 & 3.24 & & \\
\hline & & Riesgo & 55 & 3.22 & 2.15 & & \\
\hline \multirow[t]{3}{*}{ Flex. cognitiva } & Vincular & Óptimo & 22 & 2.46 & 1.87 & .25 n.s. & .029 \\
\hline & & Monitoreo & 19 & 3.63 & 2.75 & & \\
\hline & & Riesgo & 55 & 3.18 & 2.29 & & \\
\hline \multirow[t]{3}{*}{ Flex. cognitiva } & Reflexiva & Óptimo & 41 & 2.71 & 1.99 & .27 n.s. & .028 \\
\hline & & Monitoreo & 22 & 3.68 & 2.77 & & \\
\hline & & Riesgo & 33 & 3.21 & 2.34 & & \\
\hline \multirow[t]{3}{*}{ Flex. cognitiva } & CP totales & Óptimo & 30 & 2.63 & 2.17 & .23 n.s. & .031 \\
\hline & & Monitoreo & 11 & 2.64 & 2.80 & & \\
\hline & & Riesgo & 55 & 3.46 & 2.27 & & \\
\hline
\end{tabular}

Nota. a = las comparaciones post hoc muestran que en las puntuaciones altas en CP totales generalmente sus hijos/as poseen un buen desarrollo atencional; ${ }^{* *} p<.01 ; n$. s. = no significativo. 


\section{Discusión}

Los principales hallazgos de este estudio permiten identificar, por una parte, la influencia significativa de las competencias parentales de tipo formativas y protectoras de los padres, madres o cuidadores en la atención de los/as niños/as de la muestra y, por otro lado, que ninguna de las competencias parentales tuvo influencia significativa en la FC de los/as escolares.

De esta manera, a partir de los resultados obtenidos, se puede sostener que los/as niños/as cuyos padres, madres o cuidadores se encuentran en una zona de riesgo en relación con sus competencias parentales de tipo formativas y protectoras, muestran dificultades para realizar la prueba de Sendero Gris del ENFEN que involucra la habilidad de atención. En otras palabras, podríamos afirmar que aquellos padres, madres o cuidadores de la muestra que poseen escasas habilidades que promuevan el desarrollo, aprendizaje y procesos de socialización en sus hijos/as -aspectos ligados a las competencias formativas- y que, a su vez, tienen dificultades para poner en práctica aspectos de la parentalidad ligados a la protección, como el resguardo y el cuidado para un desarrollo integral de los/as hijos/as, podrían estar afectando el funcionamiento atencional de sus hijos/ as, hallazgo que, de una u otra forma, está documentado en otras investigaciones previamente señaladas (De Cock et al., 2017; Ross et al., 2019).

La influencia de las competencias formativas y protectoras en la atención de los/as niños/as, es un hallazgo bastante interesante, debido a que si bien hay investigaciones en las que se pone de manifiesto el cómo influye, por ejemplo, el estrés de las madres en el proceso de crianza en la atención de los/as niños/as, no hay estudios previos en donde se señale la importancia de las competencias parentales planteadas por Gómez y Muñoz (2014) en el sistema atencional, por lo que este estudio es un aporte y una buena contribución en este ámbito.

Del mismo modo, el hallazgo sobre la influencia de las competencias parentales formativas $y$ protectoras en la atención de los/as niños/as, está en sintonía con el estudio de Ross et al. (2019) descrito anteriormente, en el que se pone de manifiesto que las madres que presentan un mayor estrés en el proceso de crianza, y por ende tienen un menor desempeño en aspectos de la parentalidad ligados a la formación y protección de sus hijos/as, estos últimos/as son más vulnerables a los efectos del estrés agudo en tareas relacionadas a atención sostenida.

Los hallazgos de este estudio también se relacionan con la investigación que hicieron De Cock et al. (2017), en la que pudieron determinar que el estrés repercute de manera negativa en el vínculo afectivo y por ende en la interacción que tienen los padres, madres o cuidadores con sus hijos/as, quienes, a su vez, presentan, posteriormente, dificultades en su desarrollo cognitivo y funcionamiento ejecutivo general. Esto permite reflexionar en torno a las consecuencias de los episodios de estrés en el ejercicio de la parentalidad, debido a que tales situaciones podrían desencadenar en una ausencia de espacio para que los padres, madres o cuidadores compartan un tiempo significativo con sus hijos/as y en donde puedan poner en práctica las competencias parentales, las cuales son fundamentales en el desarrollo integral de los/as niños/as.

Llama la atención el hallazgo sobre la ausencia de influencia significativa de las competencias parentales en la FC de los/as niños/as de la muestra, lo que se contrapone a la evidencia científica al respecto. No obstante, esto podría ser explicado desde un punto de vista madurativo, en el sentido de que está bien documentado que el desarrollo de la flexibilidad cognitiva está modulado por la adquisición previa de otras habilidades cognitivas y, por ende, se desarrolla en etapas posteriores respecto al grupo etario de los/as niños/as de la muestra (Davidson et al., 2006; Diamond, 2013; Diamond \& Ling, 2020). Evidencia de esto es el estudio realizado por García et al. (2010), en el cual niños/as que cursaban tercero básico tuvieron un mejor desempeño en tareas que 
evaluaban la FC que los/as de primer grado. En este estudio se destaca el periodo clave de desarrollo de esta función ejecutiva, que sería entre los 6 y los 9 años, lo cual podría explicar nuestro hallazgo; ya que los/as niños/as de la muestra tienen una edad promedio de 6.79 en el caso de los niños y 6.77 en el caso de las niñas, lo que permitiría identificar que el funcionamiento ejecutivo, específicamente, la FC, aún se encuentra en etapa de desarrollo. Esto coincide con el estudio de Rodríguez et al. (2012), el cual tenía por objetivo evaluar el desarrollo evolutivo de la FC en niños/as de educación primaria. En dicho estudio se concluyó que, si bien, desde temprana edad se pueden observar ciertas FE, hay que esperar hasta la adolescencia para lograr observar un rendimiento similar al de un adulto, aspecto que los autores vinculan con los procesos madurativos que tienen lugar en la corteza cerebral en estas edades, lo cual hace predecible encontrar distintas zonas de activación en función de la edad de la muestra. En este estudio también se destaca que entre los 6 y los 9 años aún se encuentra en desarrollo la FC, mientras que entre los 9 y los 13 años ya se ejecuta de manera más consolidada, permitiendo a los/as niños/as responder acertadamente en tareas que evalúan la FC.

Lo anterior nos permite pensar que el rendimiento de los/as niños/as de la muestra en el dominio ejecutivo de la FC refleja simplemente su etapa temprana de maduración de las estructuras prefrontales, donde, principalmente, se aloja esta habilidad ejecutiva (Friedman \& Robbins, 2021; Tirapu-Ustárroz et al., 2017), más que el efecto de las competencias de sus padres/madres/cuidadores. En este sentido, creemos que si los/as mismos/as niños/as fueran evaluados/as en unos años más sus resultados en la prueba de Sendero Color podrían ser mejores. Por lo mismo, realizar un estudio longitudinal a futuro sería una de nuestras propuestas.

Como parte de las limitaciones del presente estudio, se puede considerar, en primer lugar, el tamaño de la muestra y que fue intencionada, debido a que se focalizó solo en dos escuelas, por lo que en futuras investigaciones sería importante ampliar la cantidad de participantes y analizar lo que sucede también en otras instituciones educativas. Adicionalmente, sería relevante también ampliar el rango etario de los/as niños/as de la muestra, o bien realizar investigaciones longitudinales. Esto en virtud de que como en este estudio no se halló una influencia significativa de las competencias parentales en la FC, fenómeno que podría ser explicado desde un punto de vista madurativo, sería interesante ver qué sucede al replicar el estudio considerando niños/as de edades más avanzadas o los/as mismos/as niños/as a través del tiempo. A su vez, otra limitación del estudio es haber utilizado solo las subpruebas del ENFEN Sendero Gris y Sendero Color como medidas de la atención y la flexibilidad cognitiva respectivamente, por lo que se sugiere para futuras investigaciones ampliar las pruebas de evaluación de dichos dominios cognitivos.

A pesar de estas limitaciones, los hallazgos de este estudio relevan la importancia de realizar aún más investigaciones que analicen el papel de las competencias parentales en el funcionamiento atencional de los/as niños/as, ya que si bien los resultados obtenidos en ese aspecto fueron significativos, en general hay poca información acerca de cómo impactan las competencias parentales específicas (i.e., vinculares, formativas, protectoras y reflexivas) en el sistema atencional de los/as niños/ as, por lo que sería relevante que se continuara investigando en esta misma línea para tener un mayor respaldo empírico al respecto. Lo mismo en relación con la FC, en tanto el no haber encontrado influencia de las CP sobre este dominio ejecutivo, debería animar a futuras investigaciones a profundizar en dicho hallazgo. Se podría pensar que si se replica un estudio de este tipo, con una muestra de participantes que tengan la misma edad de quienes participaron en este estudio, sea posible confirmar la explicación de tipo madurativa para la nula influencia de las CP en la FC; o bien, podría darse el caso contrario y sí 
arrojar una influencia significativa de las CP en este dominio ejecutivo, lo cual confirmaría los estudios anteriores en los que se destaca la importancia del proceso de crianza y las competencias de los padres, madres o cuidadores en el desarrollo de la FC.

A modo de conclusión, los resultados de la presente investigación permiten identificar la influencia de las competencias parentales formativas y protectoras en la capacidad de atención de los/as niños/as de la muestra. Lo anterior permite distinguir que las actitudes de los padres, madres o cuidadores que favorecen que sus hijos/as se desarrollen, aprendan y socialicen con otros/as niños/as, estimulando su aprendizaje, siendo un/a guía que les oriente y aconseje en distintas etapas de la vida, y que lleve a cabo una disciplina positiva con base en el buen trato y la preparación para vivir en la sociedad (Gómez \& Muñoz, 2014), contribuyen al rendimiento atencional de sus hijos/as, aspecto muy importante, además, para tener éxito en el sistema escolar (Bernal-Ruiz et al., 2018; Diamond, 2013).

En la misma línea, también se pudo identificar que el desarrollo de la atención en los/as niños/as estaría vinculado a las CP de tipo protectoras desplegadas por sus padres, madres o cuidadores, las que están relacionadas con las habilidades para entregar los cuidados básicos que necesitan los/as niños/as para tener seguridad física, psicosexual y emocional en los diferentes espacios y contextos en los cuales se desenvuelven, y brindar una rutina y organización de la vida diaria que les permitan disminuir el riesgo de presentar eventos de estrés infantil.

Finalmente, los resultados del presente estudio pueden servir como evidencia para el diseño de programas de crianza que promuevan la adquisición y/o el fortalecimiento de una parentalidad positiva que potencie el desarrollo socioemocional y cognitivo en los/as niños/as, entregando apoyo a las familias que presentan dificultades en sus CP y en la forma en que se relacionan con sus hijos/as.

\section{Conflicto de intereses}

Se deja constancia que, en la realización del presente estudio, no se presentó ningún conflicto de interés entre las partes involucradas.

\section{Responsabilidad ética}

Las autoras declaran que no se realizaron experimentos en este estudio ni en seres humanos ni en animales.

\section{Contribución de autoría}

Todos participaron igual y activamente en cada una de las etapas de esta investigación y en la redacción del manuscrito.

\section{Referencias}

Barudy, J., \& Dantagnan, M. (2010). Los desafíos invisibles de ser madre o padre. Manual de evaluación de competencias y resiliencia parental. Editorial Gedisa.

Baumrind, D. (1967). Child Care Practices Anteceding Three Patterns of Preschool Behavior. Genetic Psychology Monographs, 75, 43-88.

Bernal-Ruiz, F., Rodríguez-Vera, M., González-Campos, J., \& Torres-Álvarez, A. (2018). Competencias parentales que favorecen el desarrollo de funciones ejecutivas en escolares. Revista Latinoamericana de Ciencias Sociales, Niñez y Juventud, 16(1), 163-176. http:// dx.doi.org/10.11600/1692715x.16109

Bornstein, M. H. (1995). Handbook of Parenting. Lawrence Erlbaum Associates.

Bronfenbrenner, U. (1979). The Ecology of Human Development. Harvard University Press.

Caascango, K. N., Pillajo, A. I., Yépez, E. R., \& Bedón, A. N. (2020). Primera infancia: estudio relacional de estilos de crianza y las funciones ejecutivas. Revista Cognosis, 5(1), 61-78. https://doi.org/10.33936/cognosis.v5i1.2016

Comisión Nacional de Investigación Científica y Tecnológica. (2010). Declaración de Singapur sobre la integridad en la investigación. CONIYT. 
Davidson, M., Amso, D., Anderson, L., \& Diamond, A. (2006). Development of Cognitive Control and Executive Functions From 4-13 Years: Evidence from Manipulations of Memory, Inhibition, and Task Switching. Neuropsychologia, 44(11), 2037-2078. https://doi.org/10.1016/j.neuropsychologia.2006.02.006

De Cock, E., Henrichs, J., Klimstra, T. A., Janneke, B. M., Maas, A., Vreeswijk, C., Meeus, W., \& Van Bakel, H. (2017). Longitudinal Associations Between Parental Bonding, Parenting Stress, and Executive Functioning in Toddlerhood. Journal of child and family studies, 26(6), 1723-1733. https://doi.org/10.1007/s10826-0170679-7

Diamond, A. (2013). Executive Functions. Annual Review of Psychology, 64, 135-168. https://doi.org/10.1146/ annurev-psych-113011-143750

Diamond, A., \& Ling, D. S. (2020). Review of the Evidence on, and Fundamental Questions About, Efforts to Improve Executive Functions, Including Working Memory. In J. M. Novick, M. F. Bunting, M. R. Dougherty, \& R. W. Engle (Eds.), Cognitive and Working Memory Training: Perspectives from Psychology, Neuroscience, and Human Development. Oxford University Press. https://doi.org/10.1093/oso/ 9780199974467.003.0008

Fortenbaugh, F. C., DeGutis, J., \& Esterman, M. (2017). Recent Theoretical, Neural, and Clinical Advances in Sustained Attention Research. Annals of the New York Academy of Sciences, 1396(1), 70-91. https://doi.org/ 10.1111/nyas.13318

Franco-Nerín, N., Pérez-Nieto, M., \& Pérez, M. (2014). Relación entre los estilos de crianza parental y el desarrollo de ansiedad y conductas disruptivas en niños de 3 a 6 años. Psicología Clínica con Niños y Adolescentes, 1(2), 149-156.

Friedman, N. P., \& Robbins, T. W. (2021). The Role of Prefrontal Cortex in Cognitive Control and Executive Function. Neuropsychopharmacology. 47, 72-89. https://doi.org/10.1038/s41386-021-01132-0

García, A., Canet, L., \& Andrés, M. L. (2010). Desarrollo de la flexibilidad cognitiva y de la memoria de trabajo en niños de 6 a 9 años de edad. Revista Mexicana de Investigación en Psicología, 2(1), 12-19.
García, D., Zapata, A. M., \& Ortíz, J. A. (2019). Pautas de crianza y su incidencia en el desarrollo cognitivo infantil. [Tesis de pregrado, Universidad Cooperativa de Colombia]. Repositorio Institucional. https:// repository.ucc.edu.co/handle/20.500.12494/14148

Gómez, E., \& Muñoz, M. (2014). Escala de Parentalidad Positiva e2p. Fundación Ideas para la Infancia.

Hughes, C., \& Ensor, R. (2009). How do Families Help or Hinder the Emergence of Early Executive Function? En C. Lewis, \& J. I. M. Carpendale (Eds.), New Directions in Child and Adolescent Psychiatry, (pp. 35-50). https://doi.org/10.1002/cd.234

Lezak, M. D. (2004). Neuropsychologycal Assessment. Oxford University Press.

Lozano, N., Ruival, P., Riva, S., Mancilla, M., Álvarez, L., Dhers, P., Rodríguez, M., Dalmaso, M. L., Nuñez, N., \& Acquesta, M. A. (2012). Evaluación de las Funciones Ejecutivas de niños entre 6 y 12 años: Normalización de la Batería Neuropsicológica ENFEN en la zona sur de la Provincia de Buenos Aires. Hologramática, 22(2), 49-71.

Luria, A. R (1984). Atención y memoria. Martínez Roca.

Martínez, Á. C. (2010). Pautas de crianza y desarrollo socioafectivo en la infancia. Diversitas: Perspectivas en Psicología, 6(1), 111-121. https://doi.org/10.15332/ s1794-9998.2010.0001.08

Miyake, A., Friedman, N. P., Emerson, M. J., Witzki, A. H., Howerter, A., \& Wager, T. D. (2000). The Unity and Diversity of Executive Functions and their Contributions to Complex «Frontal Lobe»: A Latent Variable Analysis. Cognitive Psychology, 41(1), 49-100. https://doi.org/10.1006/cogp.1999.0734

Muñoz-Gómez, J. Á. (2019). Relación entre las dimensiones de los estilos parentales e inhibición y flexibilidad cognitiva en jóvenes de 12 años. [Tesis de maestría, Universidad Internacional de La Rioja]. https://reunir.unir.net/handle/123456789/8813

Musso, M. (2010). Funciones ejecutivas: un estudio de los efectos de la pobreza sobre el desempeño ejecutivo. Interdisciplinaria, 27(1), 95-110.

Portellano, J. A., Martínez, R., \& Zumárraga, L. (2009). ENFEN. Evaluación Neuropsicológica de las Funciones Ejecutivas en Niños. TEA ediciones. 
Rebollo, M. A., \& Montiel, S. (2006). Atención y funciones ejecutivas. Revista de Neurología, 42(2), 3-7. https:// doi.org/10.33588/rn.42S02.2005786

Ricker, T. J., Nieuwenstein, M. R., Bayliss, D. M., \& Barrouillet, P. (2018). Working Memory Consolidation: Insights from Studies on Attention and Working Memory. Annals of the New York Academy of Sciences, 1424(1), 8-18. https://doi.org/10.1111/nyas.13633

Rodríguez, C., Jiménez, J. E., Díaz, A., García, E., Martín, R., \& Hernández, S. (2012). Datos normativos para el Test de los Cinco Dígitos: desarrollo evolutivo de la flexibilidad en Educación Primaria. European Journal of Education and Psychology, 5(1), 27-38. https:// doi.org/10.30552/ejep.v5i1.74

Rojas, A. M. (2020). Factores asociados al desarrollo de las funciones ejecutivas en preescolares. [Tesis de pregrado inédita, Universidad Cooperativa de Colombia]. https://repository.ucc.edu.co/handle/ 20.500.12494/28241

Ross, L., Giuliano, R., Beauchamp, K., Berkman, E., Knight, E., \& Fischer, P. (2019). Acute Stress Impairs Children's Sustained Attention with Increased Vulnerability for Children of Mothers Reporting Higher Parenting Stress. Developmental Psychobiology, 62(4), 532-543. https://doi.org/10.1002/dev.21915

Schroeder, V., \& Kelley, M. (2010). Family Environment and Parent-Child Relationships as Related to Executive Functioning in Children. Early Child Development and Care, 180(10), 1285-1298. https://doi.org/10.1080/ 03004430902981512

Smolak, E., McGregor, K. K., Arbisi-Kelm, T., \& Eden, N. (2020). Sustained Attention in Developmental Language Disorder and Its Relation to Working Memory and Language. Journal of Speech, Language, and Hearing Research, 63(12), 1-13. https://doi.org/10.1044/ 2020_JSLHR-20-00265

Sui, J., \& Rotshtein, P. (2019). Self-Prioritization and the Attentional Systems. Current Opinion in Psychology, 29, 148-152. https://doi.org/10.1016/j.copsyc.2019.02.010
Taber, K. S. (2018). The Use of Cronbach's Alpha when Developing and Reporting Research Instruments in Science Education. Research in Science Education, 48(6), 1273-1296. https://doi.org/10.1007/s11165-016$9602-2$

The jamovi project. (2020). Jamovi (versión 1.1.9.0) [software]. JAMOVI. https://www.jamovi.org

Tirapu-Ustárroz, J., Cordero, P., Luna-Lario, P., \& HernáezGoñi, P. (2017). Propuesta de un modelo de funciones ejecutivas basado en análisis factoriales. Revista de Neurología, 64(02), 75-84. https://doi.org/10.33588/ m.6402.2016227

Tirapu-Ustárroz, J., Cordero, P., \& Bausela, E. (2018). Funciones ejecutivas en población infantil: propuesta de una clarificación conceptual e integradora basada en resultado de análisis factoriales. Cuadernos de Neuropsicología/Panamerican Journal of Neuropsychology, 12(3), 1-31. https://doi.org/ 10.7714/CNPS/ 12.3.203

Treat, A. E., Sheffield, A., Williamson, A., Hays-Grudo, J., \& Laurin, D. (2019). Adverse Childhood Experiences, Parenting, and Child Executive Function. Early Child Development and Care, 189(6), 926-937. https:// doi.org/10.1080/03004430.2017.1353978

Vargas-Rubilar, J., \& Arán-Filippetti, V. (2014). Importancia de la parentalidad para el desarrollo cognitivo infantil: una revisión teórica. Revista Latinoamericana de Ciencias Sociales, Niñez y Juventud, 12(1), 171-186. http://dx.doi.org/10.11600/1692715x.1219110813

Velarde, M. A., \& Ramírez, M. J. (2017). Efectos de las prácticas de crianza en el desempeño cognitivo en niños de edad preescolar. Revista Chilena de Neuropsicología, 12(1), 12-18.

Vera, L., \& Apolo, J. (2020). Competencias parentales: percepciones de padres de niños con discapacidad. Conrado, 16(72), 188-199. https://conrado.ucf.edu.cu/ index.php/conrado/article/view/1230

Verdejo-García, A., \& Bechara, A. (2010). Neuropsicología de las funciones ejecutivas. Psicothema, 22(2), 227-235. 
Francisca Bernal-Ruiz

Escuela de Psicología y Centro del Desarrollo en Cognición y Lenguaje CIDCL, Universidad de Valparaíso, Chile.

Doctora en Psicología con amplia trayectoria en el área de la Psicología Cognitiva y Educacional. Académica e Investigadora en universidades chilenas. Principales líneas de investigación: funciones ejecutivas y aprendizaje escolar; competencias parentales y desempeño ejecutivo en población infantil.

ORCID: https://orcid.org/0000-0001-6973-2443

Autora corresponsal: francisca.bernal@uv.cl

\section{Camila Paz Torres Pérez}

Escuela de Psicología, Facultad de Ciencias Sociales, Universidad de Valparaíso, Chile.

Licenciada en Psicología, Universidad de Valparaíso, Chile. Estudiante de 5 to año de Psicología en la Universidad de Valparaíso. Se encuentra realizando su práctica profesional en el área de Psicología Clínica en Adultos con enfoque de aceptación y compromiso.

ORCID: https://orcid.org/0000-0002-0702-8511

camila.torresp@alumnos.uv.cl

Daniela Morin Cárdenas Tapia

Escuela de Psicología, Facultad de Ciencias Sociales, Universidad de Valparaíso, Chile.

Licenciada en Psicología, Universidad de Valparaíso, Chile. Estudiante de 5 ${ }^{\text {to }}$ año de Psicología en la Universidad de Valparaíso. Se encuentra realizando su práctica profesional en el área de Psicología Clínica en Adultos con enfoque sistémico-narrativo.

ORCID: https://orcid.org/0000-0002-7126-6653

daniela.cardenast@alumnos.uv.cl

Daniela Alejandra Riveros Farías

Escuela de Psicología, Facultad de Ciencias Sociales, Universidad de Valparaíso, Chile.

Licenciada en Psicología de la Universidad de Valparaíso, Chile. Estudiante de 5 to año de Psicología de la Universidad de Valparaíso, Chile. Se encuentra realizando su práctica profesional en el área de Psicología Clínica en Adultos.

ORCID: https://orcid.org/0000-0003-2623-1897

daniela.riveros@alumnos.uv.cl

Camila Vilches Carvajal

Escuela de Psicología, Facultad de Ciencias Sociales, Universidad de Valparaíso, Chile.

Licenciada en Psicología de la Universidad de Valparaíso, Chile. Estudiante de $5^{\text {to }}$ año de la Universidad de Valparaíso. Actualmente, se encuentra realizando su práctica profesional en el ámbito de Psicología Clínica en Adultos.

ORCID: https://orcid.org/0000-0003-3024-3364

camila.vilchesc@alumnos.uv.cl

María Paz Farías Hurtubia

Escuela de Psicología, Facultad de Ciencias Sociales, Universidad de Valparaíso, Chile.

Licenciada en Psicología de la Universidad de Valparaíso, Chile. Estudiante de $5^{\text {to }}$ año de la Universidad de Valparaíso. Actualmente, se encuentra realizando su práctica profesional en el ámbito de Psicología Educacional.

ORCID: https://orcid.org/0000-0002-7420-9443

maria.fariash@alumnos.uv.cl

Linda Kim Quintana López

Escuela de Psicología, Facultad de Ciencias Sociales, Universidad de Valparaíso, Chile.

Licenciada en Psicología de la Universidad de Valparaíso, Chile.

ORCID: https://orcid.org/0000-0002-5602-1885

linda.quintana@alumnos.uv.cl 